quality of care and aligns to the principles of prudent healthcare.

- Recognition that dementia is a terminal illness and early engagement about ACP is fundamental to improving end of life care.

- Talking about ACP requires time, knowledge and sensitivity. Everyone who interacts with the person with dementia has a role to play in ensuring an ACP discussion takes place.

- A structured approach is required to prepare, equip and support health and social care professionals to progress the discussion and the equally important documentation and communication of wishes.

Good practice is an end-to-end process which is valued and understood by everyone who is part of it. It is reliable and robust; health and social care workers feel informed, supported, protected and empowered to meet the patient's wishes.

\section{PALLIATIVE RADIOTHERAPY}

1,2Linda Bedford. 'Macmillan Cancer Support, UK; ${ }^{2}$ Radiotherapy Dept., Beacon Centre, Musgrove Park Hospital, Taunton and Somerset NHS Foundation Trust, Taunton, Somerset, UK

\subsection{6/spcare-2019-mariecuriepalliativecare.17}

Introduction Radiotherapy treatment with palliative intent accounts for $35-40 \%$ of the radiotherapy department's workload. Historically, patients had a long wait to see Consultant Clinical Oncologists and receive radiotherapy for their symptoms (the most common of which is pain) (Jones et al 2014).

Service Improvement A service has been developed for rapid access to palliative radiotherapy, to improve the patient experience and access timely symptom control. This is achieved with radiographer led service where patients are seen by a Consultant Radiographer (CR), assessed for radiotherapy, consented, referred and radiation prescriptions all completed eliminating waiting times for Oncologists clinics. Including a CR led outreach service for the trust and local Hospices ensuring all patients requiring palliative radiotherapy receive timely assessment and treatment.

Aims

- Assess patients individually in terms of suitability for palliative radiotherapy.

- Promote awareness of Palliative Radiotherapy as a treatment option in managing

- metastatic cancer as a complex long term condition

- Reduction of Pill Burden (Farrell et al 2013)

- Reducing length of stay and admissions.

- Holistic pain management - liaising with palliative care teams in the community.

Results A streamlined process has been developed demonstrating continuity of care. Specialist nurses and physios now contact the CR for advice and direct referrals. Pain is managed in a timely manner.

Conclusion This work has highlighted the complex nature of palliative radiotherapy in the management of cancer as a long term condition enabling patients to access timely symptom control. There are still many challenges to overcome and this is very much work in progress.

\section{USING ROUTINE DATA TO IDENTIFY THE FACTORS THAT PLACE PATIENTS AT RISK OF NOT RECEIVING PALLIATIVE CARE}

${ }^{1}$ Peter Edmunds, ${ }^{2}$ Mirella Longo. 'Vancouver Coastal Health, Vancouver, Canada;

${ }^{2}$ University of Cardiff, Marie Curie Palliative Care Research Centre, UK

\subsection{6/spcare-2019-mariecuriepalliativecare.18}

Introduction Despite the benefits and growing acceptance that palliative care should be made available to seriously ill, frail and dying people (Kavalieratos et al. 2016, Singer et al. 2016), for some, this is still not the case. This study used historical routine data to determine the factors associatedwith not receiving palliative care.

Methods Four electronic healthcare databases (Discharge Abstract Database recording Hospital care, Emergency Department Database, PARIS community care database, Palliative Care patient registry database) were linked for all deceased patients over 2 years in a suburban population of 200,000 in Vancouver, Canada.

Results 2504 deceased individuals were identified. Of these, 2424 patients could be linked and assigned to a leading cause of death (761 cancer, 689 dementia/frailty, 974 organ failure). $67 \%$ of all patients had a record of receiving palliative care, however, almost half of these received less than 56 days of palliative care.

For people facing end of life illness, factors increasing the risk of not being offered palliative care were:

- No attachment to a general practitioner,

- Organ failure/non-malignant diagnoses,

- Males with organ failure/non-malignant diagnoses,

- Very low median household income and,

- Patients with dementia living at home.

Conclusion With the use of routine data it is possible to proactively identify and respond to patients in high-risk categories, reducing reliance on referral by clinicians. Routine data can be used to predict the palliative care needs of specific geographical areas.

\section{USING PROJECT ECHO TO MEET THE DEMAND FOR PALLIATIVE CARE EDUCATION AND SUPPORT ACROSS MULTIPLE SETTINGS}

${ }^{1}$ Janet Diffin, ${ }^{1}$ Tracey McTernaghan, ${ }^{2}$ Aine McMullan, ${ }^{2}$ Martin Hayes ${ }^{1}$ Max Watson. ${ }^{1}$ Hospice UK, UK; ${ }^{2}$ Health and Social Care Board UK

10.1136/spcare-2019-mariecuriepalliativecare.19

Background Despite increasing demand, limited resources are often available to deliver high quality palliative care education. Project ECHO is a tele-mentoring programme that could address this disparity by using video-conferencing to deliver (i) best practice guidance and education from specialists, and (ii) case-based learning with peer discussion1. Five ECHO networks in Northern Ireland delivered palliative care education and support (Cardiology/Heart Failure, District nurses, Community pharmacy, Paediatrics, Marie Curie registered nurses). Participants set the curriculum and network aims and objectives, which included increasing participant's palliative care knowledge and skills, enhancing confidence, improving collaboration, and facilitating peer support. 45 ECHO sessions were delivered across five networks with 194 participants attending $\geq 2$. 